\title{
Lorentz shear modulus of a two-dimensional electron gas at high magnetic field
}

\author{
I. V. Tokatly \\ Moscow Institute of Electronic Technology, Zelenograd, 124498 Russia \\ G. Vignale \\ Department of Physics, University of Missouri-Columbia, Columbia, Missouri 65211, USA
}

(Received 17 June 2007; published 10 October 2007)

\begin{abstract}
We show that the Lorentz shear modulus - one of the three elastic moduli of a homogeneous electron gas in a magnetic field — can be calculated exactly in the limit of high magnetic field (i.e., in the lowest Landau level) and zero frequency. Its value is $\pm \hbar n / 4$, where $n$ is the two-dimensional electron density and the sign is determined by the orientation of the magnetic field. We use this result to refine our previous calculations of the dispersion of the collective modes of fractional quantum Hall liquids.
\end{abstract}

DOI: 10.1103/PhysRevB.76.161305

PACS number(s): 73.21.-b, 73.43.-f, 78.35.+c, 78.30.-j

The collective dynamics of a two-dimensional electron gas at high magnetic fields continues to be a subject of intense interest and a source of surprises. Witness be the recent observation, by inelastic light scattering, of a new collective mode in the fractional quantum Hall liquid at filling factor $\nu=1 / 3 .^{1}$ Theoretically, the interest arises from the fact that the electrons in the lowest Landau level (LLL) exhibit an unusual kind of collective behavior (for the most recent overview of the theory of fractional quantum Hall effect, see Ref. 2). In an ordinary two-dimensional quantum liquid collective modes arise either as hydrodynamic modes (sustained by frequent collisions in local quasiequilibrium) or as collisionless modes sustained by strong self-consistent fields (in which case a generalized hydrodynamic description is possible). In both cases the frequency of the modes tend to zero at long wavelength, reflecting the underlying translational invariance of the system. By contrast, in the incompressible fractional quantum Hall liquid the collective modes have a finite frequency in the long wavelength limit. One mode at the classical cyclotron frequency $\omega_{c}=e B / m$ ( $-e$ and $m$ being the electron charge and mass-we use SI units throughout) is completely expected as a consequence of Kohn's theorem. ${ }^{3}$ The surprise comes from the low-frequency mode, which in a fractional quantum Hall liquid (e.g., the $\nu=1 / 3$ state) tends to a finite frequency $\Delta$ which scales as $e^{2} / \ell \epsilon_{b}(\ell=\sqrt{\hbar / e B}$ being the magnetic length). The gap has long been understood as a manifestation of the incompressibility of the electron liquid in the LLL, ${ }^{4,5}$ which is also responsible for the fractional quantum Hall effect. ${ }^{6}$ (The gap closes in compressible states, e.g., the $\nu=1 / 2$ state.)

The presence of the gap raises the question whether a continuum mechanics formulation (hydrodynamics or elasticity) for this type of mode is possible at all. At first sight such a formulation can only produce gapless modes. Yet the absence of low-energy excitations or other single particle excitations overlapping the energy of the collective mode suggests that a collective description of the dynamics should be possible. ${ }^{7}$ Recently, it has been found that the problem has a natural formulation as an elasticity theory in which the elastic constants are local in space, but strongly retarded in time. ${ }^{8-10}$ Let us briefly review the essential points of this formulation.

First, the equation of motion for a Fourier component of the particle current density at frequency $\omega$, in the linear approximation and in the absence of external fields (other than a magnetic field $B$ in the negative $z$ direction) is

$$
-i m \omega \mathbf{j}(\mathbf{r}, \omega)-e B \mathbf{j}(\mathbf{r}, \omega) \times \hat{\mathbf{z}}+\boldsymbol{\nabla} \cdot \overleftrightarrow{\mathbf{P}}(\mathbf{r}, \omega)=0,
$$

where $\overleftrightarrow{\mathbf{P}}(\mathbf{r}, \omega)$ is the Fourier component of the stress tensor, and $\boldsymbol{\nabla} \cdot$ denotes its divergence. The stress tensor is defined as the expectation value of the stress tensor operator $\hat{\mathbf{P}}$, which can be directly derived from the Heisenberg equation of motion for the current operator. ${ }^{11,12}$

Second, the stress tensor is expressed (in the longwavelength limit) as a linear function of the current density

$$
P_{i j}(\mathbf{r}, \omega)=-Q_{i j k l}(\omega) u_{k l}(\mathbf{r}, \omega)
$$

(we sum over repeated indices) where

$$
u_{k l}(\mathbf{r}, \omega)=\frac{i}{2 n \omega}\left[\partial_{k} j_{l}(\mathbf{r}, \omega)+\partial_{l} j_{k}(\mathbf{r}, \omega)\right],
$$

is the Fourier component of the strain tensor ( $n$ being the homogeneous density of the electron gas) and $Q_{i j k l}(\omega)$ is the Fourier component of the homogeneous tensor of elasticity - a fourth-rank tensor, which is symmetric with respect to any of the interchanges $(i j) \leftrightarrow(k l), i \leftrightarrow j$, or $k \leftrightarrow l$. In two dimensions $Q_{i j k l}(\omega)$ has at most six independent components. However, in a two-dimensional homogeneous electron liquid in the presence of a perpendicular magnetic field the six components of $Q_{i j k l}(\omega)$ can be expressed in terms of only three independent elastic moduli in the following manner: $Q_{x x, x x}(\omega)=Q_{y y, y y}(\omega)=K(\omega)+\mu(\omega), \quad Q_{x y, x y}(\omega)$ $=\mu(\omega), Q_{x x, y y}(\omega)=K(\omega)-\mu(\omega)$, and $Q_{x x, x y}(\omega)=-Q_{y y, y x}(\omega)$ $=i \omega \Lambda(\omega)$. A compact representation is

$$
\begin{aligned}
Q_{i j k l}(\omega)= & K(\omega) \delta_{i j} \delta_{k l}+\mu(\omega)\left(\delta_{i k} \delta_{j l}+\delta_{j k} \delta_{i l}-\delta_{i j} \delta_{k l}\right) \\
& +i \omega \frac{\Lambda(\omega)}{2}\left(\varepsilon_{i k} \delta_{j l}+\varepsilon_{j k} \delta_{i l}+\varepsilon_{i l} \delta_{j k}+\varepsilon_{j l} \delta_{i k}\right),
\end{aligned}
$$

where $K(\omega), \mu(\omega)$, and $\Lambda(\omega)$ are the frequency-dependent bulk modulus, the shear modulus, and the Lorentz shear modulus, respectively. ${ }^{13}$ The first two moduli, $K$ and $\mu$, are familiar from conventional elasticity theory, ${ }^{14}$ and cannot be 
calculated without detailed microscopic input (notice however that the zero frequency limit of the shear modulus must be zero in a liquid state). The Lorentz shear modulus $\Lambda$, introduced in Refs. 8-10, is a distinctive feature of the system in the presence of a magnetic field. Physically it is responsible for the force that squeezes together two oppositely directed streamlines. Formally it arises as follows: First, rotational invariance about the $z$ axis mandates that the tensor of elasticity be invariant under the transformation $x \rightarrow y$, $y \rightarrow-x$ : This enforces the identity $Q_{x x, x y}(\omega)=-Q_{y y, y x}(\omega)$ at all frequencies. Second, at zero frequency one has an additional reflection symmetry which allows us to interchange $x$ and $y$ without the minus sign: This implies $Q_{x x, x y}(0)=-Q_{y y, y x}(0)$ $=0$. In the absence of a magnetic field this symmetry persists even at finite frequency, but the magnetic field breaks it: Hence $\Lambda(\omega)$ has a finite nonzero value.

The main purpose of this Communication is to show that the zero frequency limit of the Lorentz shear modulus $\Lambda_{0}$ $\equiv-\lim _{\omega \rightarrow 0} \Lambda(\omega)$ can be calculated exactly and has the universal value $\pm \hbar n / 4$ ( + if the magnetic field is along the negative $z$ axis, - if it is along the positive $z$ axis) when all the electrons are in the lowest Landau level. The reason is that $\Lambda_{0}$ can be expressed as a Berry curvature of the ground-state wave function with respect to a homogeneous change of the metrics, just as the Hall conductivity can be expressed as a Berry curvature with respect to a homogeneous vector potential. ${ }^{15,16}$ This analogy was first noted in a paper by Avron et al. ${ }^{17}$ entitled "The viscosity of the quantum Hall liquid," in which $\Lambda_{0}$ was calculated for a noninteracting electron gas at integral filling factor, and improperly called "viscosity." Because of the nondissipative character of the dynamics we will continue to refer to $\Lambda_{0}$ as the "Lorentz shear modulus." Unlike Avron et al., who considered only noninteracting systems at integral filling factors, we will calculate the Lorentz shear modulus for an interacting electron gas in a partially occupied lowest Landau level. We will show that the calculation can be done exactly due to a special analyticity property which every wave function in the LLL enjoys. This result is far more rigorous and generally applicable than our previous theory of the collective mode ${ }^{10}$ In particular, it is not restricted to uniform states. Here we simply apply it to the equation of motion (1) and show how the dispersion of the collective modes obtained in Ref. 10 is modified by the exact treatment of $\Lambda_{0}$.

A very useful representation of the stress tensor can be given in terms of the derivative of the Hamiltonian with respect to a metric tensor $g_{i j}(\mathbf{r})\left(d s^{2}=g_{i j} d x^{i} d x^{j}\right)$ which defines a non-Euclidean geometry in the plane $\left(g_{i j}=\delta_{i j}\right.$ is the Euclidean metrics) (see, e.g., Refs. 12 and 18). This is the analogue of defining the current operator as the derivative of the Hamiltonian with respect to the vector potential. ${ }^{19}$ To this end, let us introduce the Hamiltonian of the spinless twodimensional electron gas in a metric field $g_{i j}(\mathbf{r})$ :

$$
H[\mathbf{g}]=\sum_{n} T_{n}+\frac{1}{2} \sum_{n \neq n^{\prime}} \frac{e^{2}}{\epsilon_{b} d\left(\mathbf{r}_{n}, \mathbf{r}_{n^{\prime}}\right)},
$$

where the sums run over particle indices $\left(n\right.$ and $\left.n^{\prime}\right)$,

$$
T_{n}=-\frac{1}{2 m} g^{-1 / 4}\left(\mathbf{r}_{n}\right) D_{n, i} \sqrt{g\left(\mathbf{r}_{n}\right)} g^{i j}\left(\mathbf{r}_{n}\right) D_{n, j} g^{-1 / 4}\left(\mathbf{r}_{n}\right)
$$

is the kinetic energy of the $n$th electron, $g^{i j}$ is the inverse of $g_{i j}$,

$$
D_{n, i}=\partial_{r_{n i}}+i e A_{i}\left(\mathbf{r}_{n}\right)
$$

is the kinetic momentum operator, $\mathbf{A}(\mathbf{r})$ is the vector potential, and $d\left[\mathbf{r}, \mathbf{r}^{\prime}\right]$ is the length of the geodesics connecting $\mathbf{r}$ to $\mathbf{r}^{\prime}$ in the non-Euclidean plane. We will work in the Landau gauge $\mathbf{A}(\mathbf{r})=(B y, 0)$ with periodic boundary conditions in the $x$ direction and open boundary conditions in the $y$ direction. The linear size of the system in either direction is $L$, which we take as our unit of length, $L=1$. We have also set $\hbar=1$. In these units the operator $\mathbf{D}$ is conveniently expressed as

$$
D_{x}=\partial_{x}+2 \pi i N_{L} y, \quad D_{y}=\partial_{y},
$$

where $N_{L}$ is the number of magnetic flux quanta $(h / e)$ enclosed by the system.

The stress tensor is defined as follows:

$$
P_{i j}(\mathbf{r}, t)=2\left\langle\frac{\delta H[\mathbf{g}]}{\delta g^{i j}(\mathbf{r})}\right\rangle,
$$

where the average is taken in the time-dependent quantum state and at Euclidean metric. With this definition of the stress tensor, the equation of motion (1) is exact. The basic task of the theory is to express the stress tensor as a (linear) functional of the current density so that Eq. (1) can be closed. This is a formidable task, of course, but we know that it can be carried out exactly in principle. ${ }^{20}$

As a first step in this direction we notice a small deviation from the Euclidean metrics, arising from the infinitesimal deformation $\mathbf{r} \rightarrow \mathbf{r}+\mathbf{u}(\mathbf{r})$ (u being the integral in time of the velocity field $\mathbf{j} / n)$, can be represented in the form $g^{i j}=\delta_{i j}-2 u_{i j}$ where $u_{i j}$ is the strain tensor. Then, it is not difficult to show that $Q_{i j k l}(\omega)$ has the microscopic representation $^{21}$

$$
Q_{i j k l}(\omega)=Q_{i j k l}^{\infty}+\left\langle\left\langle\hat{P}_{i j} ; \hat{P}_{k l}\right\rangle\right\rangle_{\omega},
$$

where the first term (purely real and independent of frequency) is the instantaneous derivative of $P_{i j}$ with respect to $g^{k l}$ and the second term is the Kubo formula for the stressstress response function.

It is evident from Eq. (4) that

$$
\Lambda_{0}=-\lim _{\omega \rightarrow 0} \operatorname{Im} \frac{Q_{x x x y}(\omega)}{\omega} .
$$

Then, using Eq. (10) in combination with the geometric definition of the stress tensor (9) we arrive, after well-known manipulations analogous to the ones that lead to the expression for the Hall conductivity, ${ }^{15,16}$ to the key result

$$
\Lambda_{0}=8 \times \operatorname{Im}\left\langle\frac{\partial \psi_{0}}{\partial g^{x x}} \mid \frac{\partial \psi_{0}}{\partial g^{x y}}\right\rangle,
$$

where $\left|\psi_{0}\right\rangle$ is the ground state of the Hamiltonian (5) in the presence of homogeneous metrics $g^{i j}$. This is essentially the 
formula obtained in Ref. 17 for what they call "viscosity."

In order to proceed, it is very convenient to parametrize the metric tensor as follows:

$$
g_{i j}=\frac{J}{\tau_{2}}\left(\begin{array}{cc}
1 & \tau_{1} \\
\tau_{1} & |\tau|^{2}
\end{array}\right), \quad g^{i j}=\frac{1}{J \tau_{2}}\left(\begin{array}{cc}
\mid \tau^{2} & -\tau_{1} \\
-\tau_{1} & 1
\end{array}\right),
$$

where $\tau=\tau_{1}+i \tau_{2}$ is a complex number defining the length and orientation of the $y$ axis of the distorted plane, and $J$ is the Jacobian of the coordinate transformation that is induced by deformation. The Euclidean metrics is recovered by setting $J=1, \tau_{1}=0$, and $\tau_{2}=1$. The derivatives with respect to $g^{i j}$ evaluated at the Euclidean metrics are given by

$$
\begin{gathered}
\frac{\partial}{\partial g^{x x}}=\frac{1}{2}\left(\frac{\partial}{\partial \tau_{2}}-\frac{\partial}{\partial J}\right), \quad \frac{\partial}{\partial g^{y y}}=-\frac{1}{2}\left(\frac{\partial}{\partial \tau_{2}}+\frac{\partial}{\partial J}\right), \\
\frac{\partial}{\partial g^{x y}}=\frac{\partial}{\partial g^{y x}}=-\frac{1}{2} \frac{\partial}{\partial \tau_{1}} .
\end{gathered}
$$

Notice that the derivative with respect to $g^{x y}$ is taken at constant $g^{y x}$ and vice versa. ${ }^{22}$

Inserting Eq. (14) into Eq. (12), and noting that the wave function does not depend on $J$ ( $J$ enters the homogeneous Hamiltonian as a global scale factor), we get the following representation for $\Lambda_{0}$ :

$$
\Lambda_{0}=2 \times \operatorname{Im}\left\langle\frac{\partial \psi_{0}}{\partial \tau_{1}} \mid \frac{\partial \psi_{0}}{\partial \tau_{2}}\right\rangle
$$

where the derivatives are calculated at $\tau_{1}=0, \tau_{2}=1$, and $J$ $=1$.

The calculation of $\Lambda_{0}$ is dramatically simplified if we assume that the wave function lies within the lowest Landau level. First of all, let us define "Landau levels." It is easy to see that the eigenfunctions of the kinetic energy operator (6) with a homogeneous metric and the stated boundary conditions have the form (up to a normalization constant)

$$
\psi_{l k}(x, y)=e^{2 \pi i k x} e^{i \pi \tau N_{L} \tilde{y}^{2}} H_{l}\left(\sqrt{2 \pi \tau_{2} N_{L}} \widetilde{y}\right),
$$

where $\tilde{y}=y+k / N_{L}, k$ is an integer, and $H_{l}$ is the $l$ th Hermite polynomial. [The eigenvalue is $(l+1 / 2) \omega_{c} / J$ ]. The (degenerate) states of the lowest Landau level are the ones with $l$ $=0$ and $k$ ranging from 0 to $N_{L}-1$. From this, we immediately see that any $N$-electron wave function that lies entirely in the lowest Landau level must have the form

$$
\psi\left(\mathbf{r}_{1}, \ldots, \mathbf{r}_{N}\right)=A\left(\tau_{1}, \tau_{2}\right) f\left(\xi_{1}, \ldots, \xi_{N}\right) \prod_{i=1}^{N} e^{i \pi \tau N_{L} y_{i}^{2}},
$$

where $f$ is an analytic function of the variables $\xi_{i} \equiv x_{i}+\tau y_{i}$, and therefore also of $\tau=\tau_{1}+i \tau_{2} . A\left(\tau_{1}, \tau_{2}\right)$ is the normalization constant. Unlike $f, A$ depends separately on $\tau_{1}$ and $\tau_{2}$, i.e., it is a nonanalytic function of $\tau$. [We note that the form of Eq. (17) is, in fact, independent of boundary conditions.] Hence in general the $\tau$ dependence of any LLL many-body wave function can be represented as $\psi\left(\tau_{1}, \tau_{2}\right)=A\left(\tau_{1}, \tau_{2}\right) \Phi(\tau)$, where $\Phi(\tau)$ is a holomorphic function. Making use of this fact it is possible to show that Eq. (15) simplifies as follows: ${ }^{23}$

$$
\Lambda_{0}=-\frac{\hbar}{L^{2}}\left(\frac{\partial^{2}}{\partial \tau_{1}^{2}}+\frac{\partial^{2}}{\partial \tau_{2}^{2}}\right) \ln A\left(\tau_{1}, \tau_{2}\right) .
$$

(Here we have reinstated the physical units, multiplying the result of the dimensionless calculation by the factor $\hbar / L^{2}$, which had been previously set to 1.)

The problem is finally reduced to calculating derivatives of the normalization constant. A second major simplification occurs in the thermodynamic limit, i.e., when the number $N$ of electrons tends to infinity in such a way that $N / N_{L}=\nu$ remains constant. Because $N_{L}$ tends to infinity it is evident that only values of $y \sim 1 / N_{L} \sim 0$ contribute to the normalization integral, and therefore the dependence of the analytic factor $f$ on $\tau$ (through $\xi=x+\tau y$ ) effectively disappears. Then we immediately see that the $\tau$ dependence of the normalization constant is of the form $A\left(\tau_{1}, \tau_{2}\right) \propto \tau_{2}^{N / 4}$, and use of Eq. (18) yields

$$
\Lambda_{0}=\frac{\hbar n}{4}
$$

where $n=N / L^{2}$ is the areal density of electrons. We emphasize that this result does not depend on the detailed form of the wave function.

Let us now apply the above universal result to the dispersion of collective modes in incompressible liquid states. In Ref. 10 we developed a theory of the elastic constants in the incompressible quantum Hall liquid, based on the premise that the stress-stress response function is dominated by a single pole at the frequency $\Delta$ of the $q=0$ gap. This assumption uniquely fixes the frequency dependence of the ordinary shear modulus $\mu(\omega)$ and the Lorentz shear modulus $\Lambda(\omega)$

$$
\mu(\omega)=\frac{\mu_{\infty} \omega^{2}}{\omega^{2}-\Delta^{2}}, \quad \Lambda(\omega)=\frac{\Lambda_{0} \Delta^{2}}{\omega^{2}-\Delta^{2}},
$$

while the bulk modulus $K$ remains frequency independent for any LLL state. The excitation gap $\Delta$, the bulk modulus $K$ together with $\mu_{\infty}$ and $\Lambda_{0}$ became the phenomenological parameters of the theory $\left(\mu^{\infty}\right.$ and $\Delta$ however could be determined self-consistently from sum rule arguments provided the microscopic ground state wave function is given ${ }^{10}$ ).

Inserting the above $\mu(\omega), \Lambda(\omega)$, and $K$ into Eq. (4) and this into Eqs. (2) and (1), and solving the resulting dispersion equation we get two collective modes with frequencies $\omega_{ \pm}(q)$

$$
\begin{aligned}
\frac{\omega_{ \pm}^{2}}{\Delta^{2}}= & 1+\bar{\mu}_{\infty}\left(\bar{\mu}_{\infty}+\bar{K}\right) \frac{Q^{4}}{2}-\bar{\Lambda}_{0} Q^{2} \\
& \pm \sqrt{\left[\bar{\mu}_{\infty}\left(\bar{\mu}_{\infty}+\bar{K}\right) \frac{Q^{4}}{2}-\bar{\Lambda}_{0} Q^{2}\right]^{2}+\left(\bar{\mu}_{\infty}^{2}-\bar{\Lambda}_{0}^{2}\right) Q^{4}},
\end{aligned}
$$

where $Q=q \ell, \bar{\mu}_{\infty}=\mu_{\infty} / \hbar n \Delta, \bar{K}=\bar{K} / \hbar n \Delta$, and $\bar{\Lambda}_{0}=\Lambda_{0} / \hbar n$ are the dimensionless wave vector and elastic moduli.

In the special case of a Laughlin wave function with filling factor $\nu=1 / M$, where $M$ is an odd integer larger than 1, we have shown in Ref. 10 that $\mu_{\infty} / \Delta=\hbar n(M-1) / 4$. Combining this with our new result of Eq. (19) we get $\Lambda_{0} \Delta / \mu_{\infty}$ $=1 /(M-1)$. Hence the only phenomenological parameter 


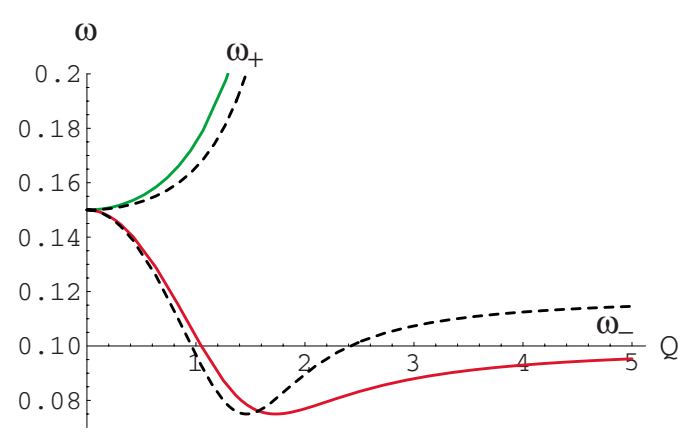

FIG. 1. (Color online) Dispersion of the collective modes at $\nu=1 / 3$ obtained from the solution of Eq. (1) for (i) the value of $\Lambda_{0}=1.4 \hbar n / 4$ used in Ref. 10 (dashed lines) and (ii) the exact value from Eq. (19) (solid lines). All other parameters are the same as in Ref. 10. Here $Q=q \ell$ and $\omega$ is in units of $e^{2} /\left(\hbar \epsilon_{b} \ell\right)$.

that is left at the current stage of the theory is the bulk modulus $K$. Importantly, $K$ is irrelevant for small $q$. Therefore at long wavelength our dispersion relations (21) for the Laughlin states are completely parameter-free.

Figure 1 shows the dispersion of the collective modes calculated at $\nu=1 / 3$ with the exact value of $\Lambda_{0}$ in comparison with the somewhat larger value of $1.4 \hbar n / 4$ used in Ref. 10 (the bulk modulus remains the same). It is clear that the overall behavior of the curve is still quite satisfactory. The reduced values of $\Lambda_{0}$ flattens the dispersion of the collective modes at large $q$ giving a shallower roton minimum. The changes in the dispersion at small $q$ are quite small.

In summary, we have presented an exact calculation of the low-frequency elasticity modulus $\Lambda_{0}$ of interacting electrons in the lowest Landau level. The fact that the calculation can be done in universal form is a consequence of two facts: (i) The existence of a geometric representation of $\Lambda_{0}$ and (ii) the analyticity of lowest Landau level wave functions with respect to the complex deformation parameter $\tau$, which describes a variation of the metrics. We believe that this general result will be useful to further elucidate the dynamics of collective states (not necessarily uniform) in the lowest Landau level.

Support from NSF Grant No. DMR-0313681 is gratefully acknowledged.
${ }^{1}$ C. F. Hirjibehedin, I. Dujovne, A. Pinczuk, B. S. Dennis, L. N. Pfeiffer, and K. W. West, Phys. Rev. Lett. 95, 066803 (2005).

${ }^{2}$ J. K. Jain, Composite Fermions (Cambridge University Press, Cambridge, UK, 2007).

${ }^{3}$ W. Kohn, Phys. Rev. 123, 1242 (1961).

${ }^{4}$ S. M. Girvin, A. H. MacDonald, and P. M. Platzman, Phys. Rev. B 33, 2481 (1986).

${ }^{5}$ V. W. Scarola, K. Park, and J. K. Jain, Phys. Rev. B 61, 13064 (2000).

${ }^{6}$ R. B. Laughlin, Phys. Rev. Lett. 50, 1395 (1983).

${ }^{7}$ S. Conti and G. Vignale, J. Phys.: Condens. Matter 10, L779 (1998).

${ }^{8}$ I. V. Tokatly, Phys. Rev. B 73, 205340 (2006).

${ }^{9}$ I. V. Tokatly, Phys. Rev. B 74, 035333 (2006).

${ }^{10}$ I. V. Tokatly and G. Vignale, Phys. Rev. Lett. 98, 026805 (2007).

${ }^{11}$ R. D. Puff and N. S. Gillis, Ann. Phys. (N.Y.) 46, 364 (1968).

${ }^{12}$ I. V. Tokatly, Phys. Rev. B 71, 165104 (2005).

${ }^{13}$ Notice that the sign of $\Lambda$ is opposite to the one used in Ref. 10 . This is to match the change in sign of the magnetic field in Eq.
(1) so that the dispersion equation for the frequencies of collective modes retains the form given in Ref. 10.

${ }^{14}$ L. D. Landau and E. M. Lifshitz, Theory of Elasticity, 3rd ed., Course of Theoretical Physics, Vol. 7 (Pergamon, Oxford, 1986).

${ }^{15}$ J. E. Avron and R. Seiler, Phys. Rev. Lett. 54, 259 (1985).

${ }^{16}$ Q. Niu, D. J. Thouless, and Y.-S. Wu, Phys. Rev. B 31, 3372 (1985).

${ }^{17}$ J. E. Avron, R. Seiler, and P. G. Zograf, Phys. Rev. Lett. 75, 697 (1995).

${ }^{18}$ C. L. Rogers and A. M. Rappe, Phys. Rev. B 65, 224117 (2002).

${ }^{19}$ M. Forger and H. Römer, Ann. Phys. (N.Y.) 309, 306 (2004).

${ }^{20}$ E. Runge and E. K. U. Gross, Phys. Rev. Lett. 52, 997 (1984).

${ }^{21}$ I. V. Tokatly, Phys. Rev. B 71, 165105 (2005).

${ }^{22}$ The factor $1 / 2$ in the last of Eqs. (14) originates from the fact that a small increment in $\tau_{1}$ changes both $g^{x y}$ and $g^{y x}$ by equal amounts, whereas in Eq. (12) only $g^{x y}$ is changed, with $g^{y x}$ remaining constant.

${ }^{23}$ For a particular case of a single particle on torus, this was noted in P. Lévay, J. Math. Phys. 36, 2792 (1995). 